\title{
Learning Curve of Aortic Arch Replacement Surgery in Chinese Mainland with Stanford Type A Aortic Dissection
}

\author{
Yuntian Sun, ${ }^{1,2}$ Yunlong Fan, ${ }^{1,2}$ Zhaorui Dong, ${ }^{1,2}$ Shixiong Wei, ${ }^{1,2}$ Chao Song ${ }^{1,2}$ \\ ${ }^{1}$ Medical School of Chinese PLA, Beijing, 100853, P.R. China; \\ ${ }^{2}$ Department of Cardiovascular Surgery, the First Medical Centre of Chinese PLA General Hospital, 100853, Beijing, China
}

\section{ABSTRACT}

Background: Stanford type A aortic dissection (TAAD) is the most common cause of death caused by aortic disease in the Chinese mainland. Patients suffering TAAD need immediate surgical treatment [Pompilio 2001; Di Eusanio 2003; Ueda 2003; Li 2013; Afifi 2016; Zhou 2019; Zhou 2021]. Emergency aortic arch replacement is difficult and risky. The prognosis following surgery varies depending on the different surgical approaches [Pompilio 2001; Kazui 2002; Di Eusanio 2003; Ueda 2003; Moon 2009; Li 2013; Afifi 2016; Zhou 2019; Zhou 2021]. Aortic arch replacement includes totalarch replacement (Sun's operation) and hemi-arch replacement. The comparative analysis of learning curves between the two procedures has not been systematically studied. In this study, we studied and analyzed the learning curves of total-arch replacement and hemi-arch replacement using cumulative sum (CUSUM) analysis.

Methods: From January 2013 to December 2019, a total of 139 Stanford TAAD operations were performed by the same surgeon and two assistants, including 61 cases of hemi-arch replacement and 78 cases of total-arch replacement. Baseline information, including preoperative conditions, intraoperative related data and postoperative prognosis, were collected. Descriptive statistics and CUSUM were used to analyze the total operation time, cardiopulmonary bypass $(\mathrm{CPB})$ time, aortic clamping (AC) time, operative mortality, incidence of postoperative complications, postoperative intensive care unit (ICU) time, hospital stay, and postoperative drainage volume.

Results: A total of 139 patients with TAAD (age $48.8 \pm$ 12.3, male, 107, female, 32) underwent emergency aortic arch replacement. A total of 61 patients $(43.9 \%)$ underwent hemi-arch replacement, and 78 patients $(56.1 \%)$ underwent total-arch replacement. The total time, cardiopulmonary bypass $(\mathrm{CPB})$ time, and aortic clamping $(\mathrm{AC})$ time of hemiarch operation were $434.2 \pm 137.0$ minutes, $243.3 \pm 87.2 \mathrm{~min}-$ utes, and $157.0 \pm 60.2$ minutes. The total, $\mathrm{CPB}$, and $\mathrm{AC}$ times of total-arch operation were $747.8 \pm 164.3$ minutes, $476.4 \pm$ 121.6 minutes, and $238.5 \pm 67.6$ minutes. The mortality of

Received November 7, 2021; accepted November 19, 2021.

Correspondence: Dr. Chao Song, Department of Cardiovascular Surgery, Chinese PLA general Hospital, 100853, Beijing, China (e-mail: 617125202@qq.com). hemi-arch operation was $3.3 \%$, and that of total-arch operation was $6.4 \%$. The incidence of complications after hemiarch operation was $11.3 \%$, and that after total-arch operation was $46.2 \%$. The ICU time and hospital stay after hemi-arch surgery were $7.3 \pm 4.4$ days and $27.2 \pm 16.2$ days, respectively, and the ICU time and total hospital stay after total-arch surgery were $7.2 \pm 5.9$ days and $24.0 \pm 10.3$ days, respectively. The total drainage volume after hemi-arch operation was 2182.4 $\pm 1236.4 \mathrm{ml}$, and that after total-arch operation was $2467.3 \pm$ $1385.7 \mathrm{ml}$. According to CUSUM analysis, the same cardiovascular surgery team seems to have different learning curves in the time of two operations. CUSUM analysis of intraoperative and postoperative indicators shows that after a certain period of professional and systematic cardiovascular surgery training, aortic hemi-arch replacement has the characteristics of short learning cycle and easy to master for surgeons, while total-arch replacement requires a longer learning cycle.

Conclusions: Although the emergency operation of TAAD is difficult and risky, according to results the of CUSUM analysis, cardiovascular surgeons can achieve better learning results in hemi-arch replacement than total-arch replacement.

\section{INTRODUCTION}

The occurrence of aortic dissection typically has been considered to be due to the blood flowing through the torn intima from the true lumen to the false lumen [Sherifova 2019], and TAAD could also quickly lead to death from not enough blood flow to the heart or rupture of the aorta [Wang 2020]. Delayed medical intervention will lead to increased mortality, the mortality of the disease will increase by $1 \%$ every 1 $\mathrm{h}$ since the onset, and mortality will be in the range of $30 \%$ to $50 \%$ within $48 \mathrm{~h}$ [Braverman 2011; Sun 2011; Li 2013]. With the improvement in early imaging diagnosis technology, hemostatic ability of artificial vascular materials, cardiopulmonary bypass technology and neuroprotection technology, the operative mortality of aortic dissection has decreased from 40\% reported by DeBakey to about 8\% [DeBakey 1965; DeBakey 1966; DeBakey 1982; Pompilio 2001; Di Eusanio 2003; Ueda 2003; Li 2013; Afifi 2016; Wang 2021].

In the field of cardiovascular surgery in the Chinese mainland, the ascending aorta plus hemi-arch replacement has been proposed for TAAD [Moon 2009]. Meanwhile total aortic arch replacement and descending aortic stenting (Sun's 
operation) have also been considered as an alternative available treatment, which could significantly reduce the incidence of distal anastomotic bleeding and has a high long-term false lumen occlusion rate [Sun 2011; Ma 2013]. However, surgery involving aortic arch is technically demanding and complex, especially for young inexperienced cardiovascular surgeons.

Cumulative sum (CUSUM) analysis method could be used to prove the specificity of learning cycle of surgical team through clinical perioperative complications and mortality [He 2021; Kudsi 2021; Toennesen 2021]. By accumulating the clinical data information of each patient in a treatment, the CUSUM analysis accumulate the biased data to achieve the amplification effect, and the improved sensitivity of detecting the biased data in the treatment has been achieved. In recent years, CUSUM has been more and more widely used in the analysis of clinical surgery, while few studies involving macrovascular diseases [Liu 2020; Braca 2021; He 2021; Kudsi 2021; Lee 2021; Lenfant 2021; Szymczak 2021; Toennesen 2021; Verhaeghe 2021]. And the comparison of learning curve effect between hemi-arch replacement and total-arch replacement in the treatment of TAAD has not been systematically studied.

As one of the major cardiovascular centers in the Chinese mainland, TAAD repair has been performed as a kind of routine procedure in our center. From January 2013 to December 2019, a total of 139 emergency aortic arch replacement procedures have been performed in our center by the same surgeon as well as two assistants. So far, more than 2000 heart transplantation, coronary heart disease, valve disease, large blood vessels, and minimally invasive heart surgeries have been completed by this surgeon. A comprehensive database has been established by us regarding TAAD repair. In this study, the CUSUM analysis was used to evaluate the learning curve of two kinds of aortic arch replacement surgery in patients with TAAD based on the above data, and its effectiveness and reliability for the learning experience of cardiovascular surgery team also were further analyzed.

\section{METHODS}

Study population and preoperative evaluation: From January 2013 to December 2019, a total of 178 emergency aortic arch replacement procedures, including aortic hemiarch and total-arch replacement, were performed in our center by the same surgical team. Patients suffering acute coronary syndrome, recent stroke, cardiac arrest, poor splanchnic organ perfusion, hypoxemia requiring mechanical ventilation and previous history of cardiac surgery were excluded. A total of 139 patients eventually were included in the study. The entire study cohort was divided into two groups, including the hemi-arch replacement group and total-arch replacement group. Baseline information, including preoperative conditions, intraoperative related data and postoperative prognosis of the two groups, systematically were compared and analyzed.

Surgical procedure: Under the condition of hypothermic circulatory arrest (DHCA), ante-grade cerebral perfusion was performed in all cases, and ice caps were used to strengthen brain protection. During operation, bilateral cerebral oxygen saturation routinely was monitored by cerebral oxygen saturation monitor. The patient took the supine position, and we cut through the midpoint of the right subclavian to the axillary to expose the right axillary artery. After heparinization, it was anastomosed with the artificial blood vessel, and then connected with the artery tube of cardiopulmonary bypass. We adopted anterior median thoracotomy, inserted a bicaval intravenous cannula through the right atrium to establish cardiopulmonary bypass, placed a left cardiac drainage tube through the right upper pulmonary vein, clamped and cut the ascending aorta, and perfused HTK cardioplegia through the openings of the left and right coronary arteries. Transection was performed at about $3 \mathrm{~cm}$ above the junction of the sinus canal, the ascending aorta was explored, location of the breach was determined, and the aortic valve annulus and leaflet structure were examined. Aortic sinus plasty was performed after transection at about $5 \mathrm{~mm}$ above the junction of the sinus canal. Cool down to the anal temperature of $21^{\circ} \mathrm{C}$ and the nasopharyngeal temperature of $18.5^{\circ} \mathrm{C}$. Free the upper three branches of the aortic arch and set a blocking band, blocked the roots of the three blood vessels, opened the ascending aortic blocking forceps, continue cerebral perfusion through the axillary artery, and started lower body circulatory arrest. Before total-arch replacement, the elephant trunk stent was implanted into the descending aorta, and the proximal end of the stent was located at the proximal end of the left common carotid artery at the aortic arch. We repaired the proximal end of the arch and reshaped it. Disconnected the three arteries above the aortic arch, anastomosed the two branches of the four artificial vessels with the left subclavian artery and the left carotid artery, respectively, blocked the two segments of the artificial vessel with vascular blocking forceps, connected the other integrated external circulation artery tube with the branch of the artificial vessel for perfusion, and performed bilateral carotid cerebral perfusion. The proximal end of the aortic arch was reconstructed and reshaped again, anastomosed with the main artificial blood vessel, completely vented, the distal end of the artificial blood vessel was opened, and lower body perfusion was restored. The proximal end of the artificial blood vessel was properly constructed and anastomosed with the residual end of the ascending aorta. Finally, we anastomosed the last branch of the four-branch artificial blood vessel with the innominate artery and completely exhausted the air at the same time. Rewarming began after venous oxygen saturation was greater than $80 \%$. After the heart returned to beating, it gradually returned to sinus rhythm. After rewarming to anal temperature of $34^{\circ} \mathrm{C}$, cardiopulmonary bypass was stopped gradually, and ventricular systolic motion and valve function were evaluated by transesophageal ultrasound. We stopped bleeding thoroughly, neutralized heparin with protamine, and removed the cardiopulmonary bypass intubation. The artificial blood vessel of right axillary artery was ligated, the chest was closed layer by layer, and wound was sutured layer by layer. Above, we describe the surgical process of total aortic arch replacement, while hemi-arch replacement is simpler. It 
only needs to remove the small, curved side of the aortic arch after opening the ascending aorta blocking forceps, build the arch and plastic, and replace the hemi-arch after appropriate construction of artificial blood vessels.

Data collection: The baseline information, including preoperative basic characteristics, intraoperative and postoperative data of all patients, were collected from the cardiovascular surgery database of PLA general hospital.

Study endpoints: The main learning end point of this study was the total operation time. Secondary end points included cardiopulmonary bypass time, aortic clamping time, postoperative ICU days, total hospital stay, postoperative total drainage volume, postoperative complication rate, and operative mortality.

Postoperative complications were defined as postoperative complications without preoperative complications, such as stroke, pericardial tamponade, artificial vessel rupture, arrhythmia, nervous system complications, acute liver, and kidney dysfunction caused by organ ischemia, etc. Operative mortality was defined as hospital death or death within 30 days after surgery.

Statistical analysis: The CUSUM analysis in minitab19.0 software was used to analyze the data, so as to evaluate the learning curve of hemi-arch replacement and total-arch replacement for TAAD. CUSUM is a time-weighted control chart method. It calculates the deviation degree between the observed value and target value of each sample and calculates the cumulative sum by summation. In our study, the difference between the sample observation value of each patient in chronological order and the average target value of the whole group of patients was cumulatively summed to obtain the learning curve value. The calculation formula of the learning curve evaluation index is $a=X_{i}-X_{0}$; $a$ is the quantitative value of surgical indicators. $X_{0}$ is the probability that the evaluation index fails to reach the target value, and $X_{i}$ represents whether each operation reaches the target value. When the surgical evaluation index reaches the target value, $X_{i}=0$. If the target value is not reached, $X_{\mathrm{i}}=1$. Draw the learning curve based on the cumulative summation value and fit the polynomial curve. When the curve slope $\mathrm{k}$ changes from positive to negative, it will successfully cross the learning curve. The data were statistically analyzed, and the fitting curve was established by spss 23.0. The measurement data of normal distribution are expressed as mean \pm standard deviation, and two independent sample t-test is used for comparison. Rank sum test was used for comparison between non-normal distribution measurement data groups. Chi square test was used to compare the counting data, with $P<0.05$ indicating that the difference was statistically significant.

\section{RESULTS}

Demographics and baseline characteristics: From January 2013 to December 2019, the center received 178 patients with TAAD, of whom 139 underwent emergency aortic arch replacement (age $48.8 \pm 12.3$, male, 107, female, 32). Hemiarch replacement was performed in 61 cases and total-arch replacement in 78 cases. According to past medical history, 80 patients $(57.6 \%)$ had hypertension, and 70 patients $(50.4 \%)$ had diabetes. According to relevant examination results and genetic consultation, 13 patients $(9.4 \%)$ were considered to have Marfan syndrome. The degree of aortic regurgitation was more than mild in 86 cases $(61.9 \%)$. Except that the age of hemi-arch replacement group was higher than that of total-arch replacement group $(P<0.05)$, there was no significant difference in other basic characteristics. (Table 1)

Procedural and postoperative outcomes: Among 139 patients who underwent aortic surgery, 61 cases $(43.9 \%)$ underwent hemi-arch replacement, and 78 cases (56.1\%) underwent total-arch replacement. In terms of intraoperative indexes, the total operation time, cardiopulmonary bypass time and aortic clamping time in the hemi-arch replacement group were $434.2 \pm 137.0 \mathrm{~min}, 243.3 \pm 87.2 \mathrm{~min}$, and $157.0 \pm$ $60.2 \mathrm{~min}$, respectively. The total operation time of total-arch replacement group was $747.8 \pm 164.3 \mathrm{~min}$, cardiopulmonary

Table 1. Demographics and baseline characteristics

\begin{tabular}{|c|c|c|c|c|}
\hline Male & $43(70.5)$ & $64(82.0)$ & $107(77.0)$ & 0.108 \\
\hline BMI & $26.6 \pm 3.9$ & $26.3 \pm 4.0$ & $26.4 \pm 3.9$ & 0.718 \\
\hline Smoke & $37(60.7)$ & $54(69.2)$ & $91(65.5)$ & 0.291 \\
\hline Hypertension & $35(57.3)$ & $45(57.7)$ & $80(57.6)$ & 0.970 \\
\hline Diabetes & $30(49.2)$ & $40(51.3)$ & $70(50.4)$ & 0.806 \\
\hline Marfan syndrome & $5(8.2)$ & $8(10.3)$ & $13(9.4)$ & 0.679 \\
\hline Aortic regurgitation $(>2+)$ & $37(60.7)$ & $49(62.8)$ & $86(61.9)$ & 0.794 \\
\hline
\end{tabular}

*Age in the hemi-arch replacement group was significantly higher than the other group 
bypass time was $476.4 \pm 121.6 \mathrm{~min}$, and aortic clamping time was $238.5 \pm 67.6 \mathrm{~min}$. There was a significant difference between the two groups at these three times $(P<0.05)$. In terms of postoperative indexes, there was no significant difference between the two groups except for the incidence of postoperative complications $(P<0.05)$. Details of intraoperative and postoperative results are shown in Table 2. (Table 2)

Fitting curves of key indicators: According to the collected patient data of key intraoperative indicators (total operation time, $\mathrm{CPB}$ time, $\mathrm{AC}$ time), the corresponding fitting curve is established by SPSS software. We select the correct fitting curve through the value of $R^{2}$, because when $P<0.05$, the fitting is judged to be successful, and the $R^{2}$ value judges the fitting degree. The closer the $R^{2}$ value is to 1 , the better the fitting degree is. The equations of the fitting curves of the hemi-arch replacement group at three times are $\mathrm{Y}=368.7847217330303$ * $0.9845546379211212^{* *} \mathrm{x}\left(\mathrm{R}^{2}=0.540\right), \mathrm{Y}=250.774545767489$ * $0.9824435691951127^{* *} \mathrm{x}\left(\mathrm{R}^{2}=0.557\right), \mathrm{Y}=650.0974407629664$ * $0.9853917144722686^{* *} \times\left(R^{2}=0.611\right)$. The equations of fitting curves for three times of total-arch replacement group are $\mathrm{Y}=575.6139169285684$ * $0.9943417419120085^{* *} \mathrm{x}\left(\mathrm{R}^{2}=0.223\right)$, $\mathrm{Y}=265.0816241936741$ * $0.9962866264940706^{* *} \mathrm{x}\left(\mathrm{R}^{2}=0.082\right)$, $\mathrm{Y}=879.1498066102731$ * $0.9952777368326483^{* *} \mathrm{x}\left(\mathrm{R}^{2}=0.218\right)$. According to the assumed shape of the fitting curve, it can be seen that the trend of total operation time, CPB time and $\mathrm{AC}$ time in the hemi-arch replacement group has a relatively steep slope, which indicates that the learning period is more rapid. From the value of $R^{2}$, we can see that the hemi-arch replacement group has better fit than the total-arch replacement group. (Figure 1) (Figure 2)

CUSUM analysis: We collected the intraoperative key indexes (total operation time, CPB time and AC time) of hemi-arch replacement group and total-arch replacement group and linked them with the number of cases for CUSUM analysis. (Figure 3) (Figure 4) We found that when the number of operation cases reached 40, the learning curve reached the peak and there was an obvious turning point, so the operation crossed the learning curve after 40 cases. When the number of total-arch replacement reached 69 cases, the learning curve reached the peak, so the operation crossed the learning curve after 69 cases. Taking the peak as the boundary, the learning curve is divided into two periods. The first period is the learning improvement period $(1 \sim 40$ cases of hemi-arch and $1 \sim$ 69 cases of total-arch), and the second period is the mastery period ( $41 \sim 61$ cases of hemi-arch and $70 \sim 78$ cases of totalarch). First, the age, gender, body mass index (BMI), smoking history, drinking history, history of hypertension, history of diabetes, history of Marfan syndrome, and aortic valve regurgitation were compared between the two groups at two periods. (Table 3) (Table 4) The basic characteristics of the two periods of hemi-arch replacement group were no different except for diabetes history and aortic valve regurgitation $(P<$ $0.05)$. There was no difference in the basic characteristics of the two periods in the total-arch replacement group except the history of smoking and Marfan syndrome $(P<0.05)$. Secondly, the total operation time, $\mathrm{CPB}$ time, $\mathrm{AC}$ time, postoperative ICU time, hospital stay, postoperative drainage volume, incidence of postoperative complications, and operative mortality of the two groups were compared. (Table 5) (Table 6) The evaluation indexes of second period operation in hemi-arch replacement group were analyzed. While there was no difference in mortality $(P>0.05)$, the second period was significantly better than the first period. Except for aortic clamping time, ICU time and total hospital stay, there was no significant difference between the two periods in the total-arch replacement group $(P>0.05)$. The other indexes in the second period were significantly better than those in the first period.

\section{DISCUSSION}

Aortic dissection is a serious clinical cardiac macrovascular disease, among which Stanford type A dissection is a more serious type, and its disability rate and mortality rate

Table 2. Procedural and postoperative outcomes

\begin{tabular}{lccc}
\hline Outcomes & Hemi-arch replacement $(N=61)(43.9)$ & Total-arch replacement $(N=78)(56.1)$ & Total $(N=139)(100)$ \\
\hline Total-operative (min)* & $434.2 \pm 137.0$ & $747.8 \pm 164.3$ & $610.2 \pm 218.2$ \\
CPB (min)* & $243.3 \pm 87.2$ & $476.4 \pm 121.6$ & $374.1 \pm 158.2$ \\
AC (min)* & $157.0 \pm 60.2$ & $238.5 \pm 67.6$ & $202.8 \pm 76.0$ \\
ICU stay (days) & $7.3 \pm 4.4$ & $7.2 \pm 5.9$ & $0.000^{*}$ \\
Hospital stay (days) & $27.2 \pm 16.2$ & $24.0 \pm 10.3$ & $0.000 *$ \\
Postoperative drainage & $2182.4 \pm 1236.4$ & $2467.3 \pm 1385.7$ & $25.4 \pm 13.3$ \\
volume (ml) & $7(11.3)$ & $36(46.2)$ & $2342.2 \pm 1345.2$ \\
Complications** & $2(3.3)$ & $5(6.4)$ & 0.300 \\
Operative mortality & & $43(30.9)$ & $7(5.0)$ \\
\hline
\end{tabular}

\footnotetext{
*The mean total-operative time of CPB and $\mathrm{AC}$ in the hemi-arch replacement group was significantly different from the total-arch replacement group.

**The incidence of postoperative complications in the hemi-arch replacement group was significantly lower than in the total-arch replacement group.
} 

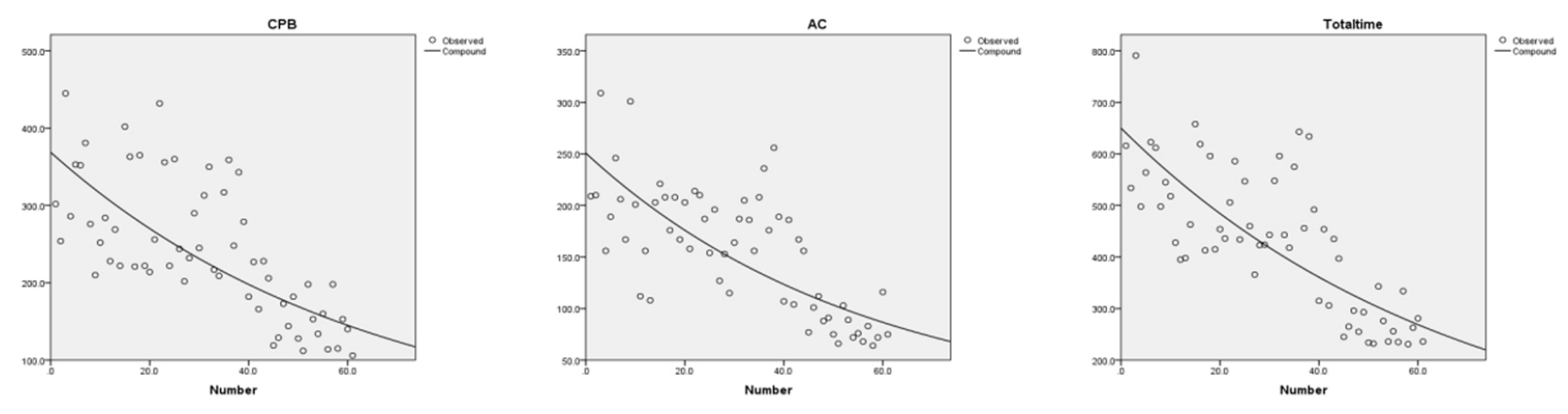

Figure 1. Hemi-arch replacement group
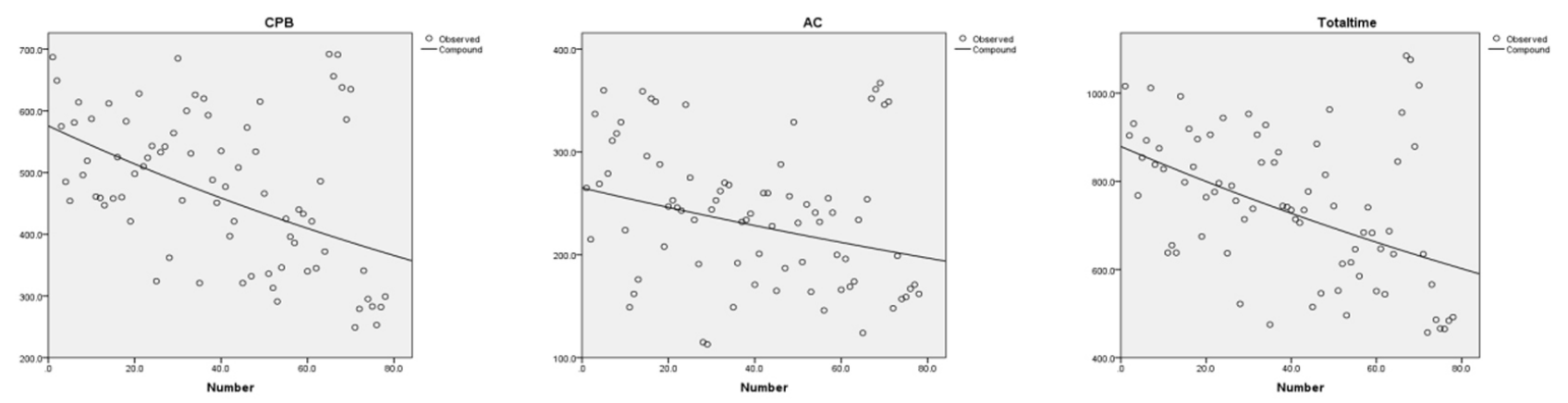

Figure 2. Total-arch replacement group
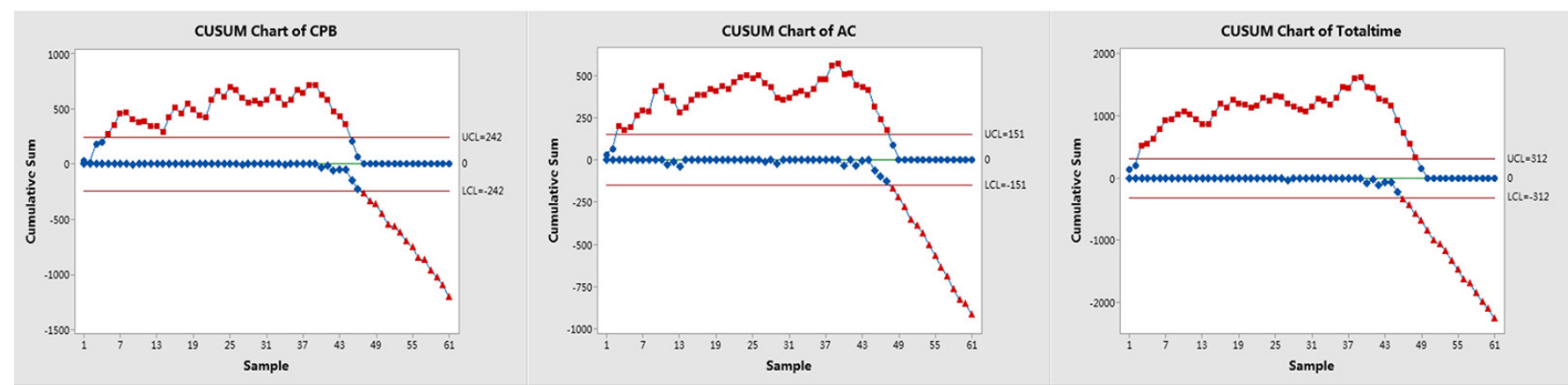

Figure 3. Hemi-arch replacement group
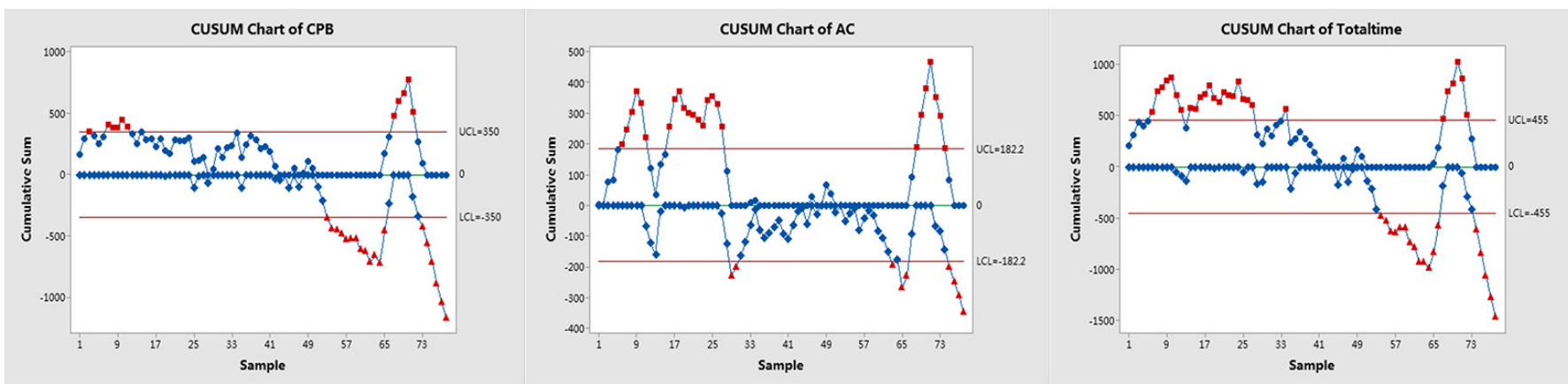

Figure 4. Total-arch replacement group 
Table 3. The demographics and baseline characteristics of hemi-arch replacement

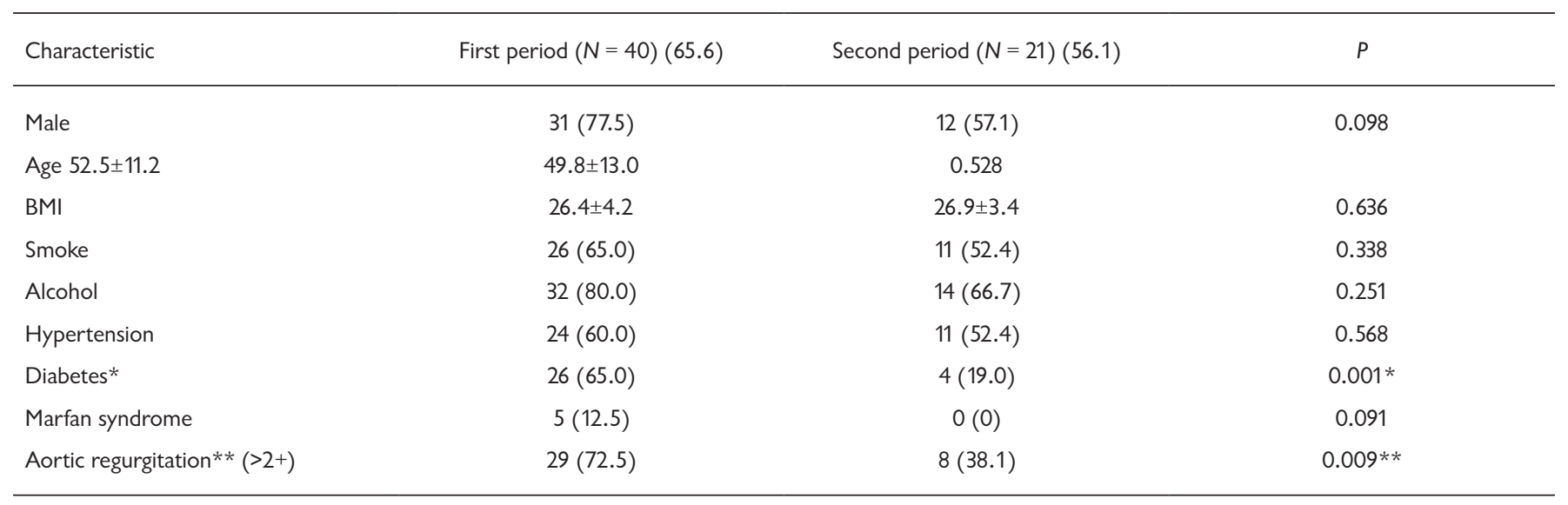

*Diabetes in group "first period" was significantly higher than the other group.

**Aortic regurgitation in group "first period" was significantly higher than the other group.

Table 4. The demographics and baseline characteristics of total-arch replacement

\begin{tabular}{|c|c|c|c|}
\hline Male & $56(81.1)$ & $8(88.9)$ & 0.570 \\
\hline BMI & $26.4 \pm 4.0$ & $25.2 \pm 3.4$ & 0.450 \\
\hline Smoke* & $45(65.2)$ & $9(100.0)$ & $0.033 *$ \\
\hline Hypertension & $40(58.0)$ & $5(55.6)$ & 0.890 \\
\hline Diabetes & $38(55.1)$ & $2(22.2)$ & 0.064 \\
\hline Marfan syndrome** & $5(7.2)$ & $3(33.3)$ & $0.015 * *$ \\
\hline Aortic regurgitation $(>2+)$ & $45(65.2)$ & $4(44.4)$ & 0.225 \\
\hline
\end{tabular}

*Smoke in group "first period" was significantly higher than the other group.

**Marfan syndrome in group "first period" was significantly higher than the other group.

are quite high [DeBakey 1965; DeBakey 1966; DeBakey 1982; Pompilio 2001; Di Eusanio 2003; Ueda 2003; Li 2013; Afifi 2016; Zhou 2019; Li 2020; Liu 2020; Wang 2021; Zhou 2021]. Ascending aorta and hemi-arch replacement typically have been used as the main treatment [DeBakey 1965; DeBakey 1966; DeBakey 1982; Moon 2009]. In 1996, Kato et al. reported the technique of reconstructing the ascending aorta and aortic arch with four branches of blood vessels and inserting vascular stents into the descending aorta to treat TAAD [Kato 1996]. On this basis, Professor Li Zhong Sun proposed Sun's operation [Sun 2011]; the technique uses a composite 4-branched graft for total aortic arch replacement, and a specially designed frozen elephant trunk stent is implanted in the descending aorta. He believes this new operation cannot only significantly reduce the operative mortality, but also reduce the risk of postoperative complications, such as aneurysms and thrombosis. At present, many scholars in the Chinese mainland support his view and recommend this new procedure as the standard procedure for TAAD [Sun 2011; Ma 2013; Luo 2021]. Some literature report no significant difference in aortic complications or re intervention rate between total aortic arch replacement and hemi-arch aortic arch replacement [Kim 2011; Poon 2016]. This is different from the results of our study.

In recent years, very few studies regarding CUSUM learning curve of emergency aortic arch replacement in the treatment of TAAD have been reported, and there is basically no comparative study on human factors of hemi-arch replacement and total-arch replacement by CUSUM integral method. Liu et al. showed that three cardiovascular surgeons completed total aortic arch replacement [Liu 2020]; the learning curve of two of them peaked in about 10 cases and 75 cases, but his study chose different surgical teams. It was analyzed by grouping method, which in turn resulted in subjective and 
Table 5. The procedural and postoperative outcomes of hemi-arch replacement

\begin{tabular}{lccc}
\hline Outcomes & First period $(\mathrm{N}=40)(65.6)$ & Second period $(\mathrm{N}=21)(56.1)$ & $P$ \\
\hline Total operative $(\mathrm{min}) *$ & $509.6 \pm 98.4$ & $290.6 \pm 66.8$ & $0.000^{*}$ \\
CPB (min)* & $288.9 \pm 69.0$ & $156.4 \pm 38.1$ & $0.000 *$ \\
AC (min)* & $188.5 \pm 40.1$ & $97.2 \pm 34.1$ & $0.000 *$ \\
ICU stay (days)\# & $8.2 \pm 4.6$ & $5.7 \pm 3.6$ & $0.005 \#$ \\
Hospital stay (days)\# & $31.9 \pm 18.1$ & $18.4 \pm 5.3$ & $0.000 \#$ \\
Postoperative drainage volume $(\mathrm{ml}) \# \#$ & $2553.9 \pm 1241.2$ & $1474.8 \pm 883.3$ & $0.000 \# \#$ \\
Complications** & $7(17.5)$ & $0(0)$ & $0.042 * *$ \\
Operative mortality & $2(5.0)$ & $0(0)$ & 0.297 \\
\hline
\end{tabular}

*The mean time of total-operative, CPB and AC in the "first period" group was significantly different from the "second period" group.

**The incidence of postoperative complications in the "first period" group was significantly higher than in the "second period" in group.

\#The days of ICU and hospital stay in the "first period" group was significantly higher than in the "second period" in group.

\#\#The postoperative drainage volume in the "first period" group was significantly higher than in the "second period" in group.

Table 6. The procedural and postoperative outcomes of total-arch replacement

\begin{tabular}{lccc}
\hline Outcomes & First period $(N=69)(88.5)$ & Second period $(N=9)(11.5)$ & $P$ \\
\hline Total operative $(\mathrm{min})^{*}$ & $771.9 \pm 147.1$ & $563.2 \pm 180.2$ & $0.001 *$ \\
CPB (min)* & $496.3 \pm 107.6$ & $285.1 \pm 28.8$ & $0.000^{*}$ \\
AC (min) & $242.7 \pm 65.1$ & $206.4 \pm 81.2$ & 0.053 \\
ICU stay (days) & $7.3 \pm 6.2$ & $6.2 \pm 3.5$ & 0.850 \\
Hospital stay (days) & $24.4 \pm 10.4$ & $21.3 \pm 9.6$ & 0.316 \\
Postoperative drainage volume $(\mathrm{ml})^{* *}$ & $2622.1 \pm 1375.0$ & $1280.0 \pm 786.1$ & $0.001 * *$ \\
Complications\# & $34(49.3)$ & $2(22.2)$ & $0.000 \#$ \\
Operative mortality & $5(7.2)$ & $0(0.0)$ & $0.01 \#$ \\
\hline
\end{tabular}

*The mean time of total operative and CPB in the "first period" group was significantly different from the "second period" group.

**The postoperative drainage volume in the "first period" group was significantly higher than the "second period" in group.

\#The incidence of postoperative complications in the "first period" group was significantly higher than the "second period" in group.

\#\#The incidence of postoperative complications in the "first period" group was significantly higher than the "second period" in group.

biased results. Moreover, the length of the learning cycle of total-arch replacement is significantly related to the operator's experience and learning ability. Our study chose the same surgical team, which can avoid the influence of factors other than the surgery itself to the greatest extent.

In our study, the total operation time, cardiopulmonary bypass time, aortic clamping time, operative mortality, incidence of postoperative complications, postoperative intensive care time, hospital stay, and postoperative drainage were used as evaluation measures. CUSUM method was used to draw the learning curve to analyze the effects of two different surgical methods in the treatment of TAAD. According to the statistical results, our cardiac surgery team successfully crossed the learning curve after 40 cases of hemi-arch replacement. The total operation time, cardiopulmonary bypass time and aortic clamping time was significantly decreased, while the surgical technology was significantly improved, and the learning curve could be crossed after the completion of the 69th procedure. Through the changes of intraoperative and postoperative data of the two surgical methods in the two periods, we found that the progress of hemi-arch replacement was more obvious, in terms of aortic clamping time, ICU days, and total hospital stay. Compared with hemi-arch replacement, the difference in total arch replacement mainly was reflected in mortality. In the field of cardiac surgery, the total operation time is very important to the prognosis of postoperative patients, and it also reflects the operator's proficiency in the operation to a great extent. According to the results of preoperative case discussion and the analysis of postoperative causes of death, we found that total-arch replacement is complex and difficult, while patients with aortic dissection are usually in a dangerous condition, so it is necessary to accurately judge the location 
of the breach and eliminate the false lumen in time. Growing surgeons need a long operation time to carry out Sun's operation in the early period, and too long an operation time often brings serious complications. Of course, the perfect learning curve is not achieved easily for the surgical team. Crossing the turning point of the learning curve does not mean fully mastering the technology. Sometimes, it also is affected by the patient's condition, resulting in great differences. In short, the learning curve of total-arch replacement has the characteristics of long-time span, tortuous growth process, and high requirements for the early level of the operator team.

The advantage of total-arch replacement (Sun's operation) is that the unique stent used has good flexibility and durability [Sun 2011]. Since Sun's operation was popularized, it has achieved a good reputation in the Chinese mainland [Sun 2011; Ma 2013]. However, the process of aortic arch repair is complex, and there is an uncertain risk of stent entering the descending aorta. It still is a great challenge for many young or inexperienced cardiovascular surgeons. Although the hemi-arch replacement operation takes a long time, the operation process is simpler, and the operation time is shorter compared with total-arch replacement. This kind of operation is relatively easy for cardiovascular surgeons in their growth period to master and has a short learning cycle. Since 2003, Sun's operation continuously has been improved, while there still are some deficiencies. Tamai et al. reported a case of death caused by the accidental insertion of elephant trunk stent into the false lumen [Tamai 2020], and concluded that even if the chief surgeon is an experienced macrovascular surgeon, there are individual differences in some patients, such as a large breach at the beginning of the descending aorta and a small true lumen. The bending shape and radian of elephant trunk stent mainly depend on the experience and hand feeling of the surgeon, so it is possible to implant the distal end of the stent through a large breach into the false lumen. For lack of obvious sense of resistance, this phenomenon is difficult to find in time, resulting in the death of patients. In the discussion of death cases after total-arch replacement in our center, although there was no case that the elephant trunk stent mistakenly entered the false lumen, there were other problems. For example, due to the difficulty and complexity of operation, the average operation time is too long, which will lead to a long time of organ ischemia, serious disorder of coagulation system, and multiple organ failure in the state of circulatory arrest in the lower body. Finally, the patient's condition could not be reversed, and in turn resulted in death. On the other hand, although total-arch replacement employed strategies, such as selective cerebral perfusion and hypothermic circulatory arrest to protect the brain, the circulatory arrest time is too long, resulting in neurological complications, such as delirium, coma, drowsiness, limb numbness, and slow recovery caused by cerebral ischemia and hypoxia. At this point, hemi-arch replacement has obvious advantages.

Our research has some shortcomings. First, the average age of our subjects was $48.8 \pm 12.3$ years, which was younger than the TAAD study cohort reported in the West [Moon 2009; Kim 2011; Poon 2016], and thus confounding factors caused by age and regional factors may be an unavoidable.
Second, this study is limited to the surgical team led by a chief surgeon, who has rich experience in cardiovascular surgery. Although the confounding factor of experience is removed, which makes the results more likely to prove the effectiveness of this operation, for inexperienced surgeons, this research result is not well representative, which should be considered in our follow-up research. Finally, due to the short time of total-arch replacement (Sun's operation) in our center and the insufficient number of cases involved in the study, there are some uncertain results in the learning curve of this study, so there is a certain bias.

In a word, CUSUM could be used to further analyze the sample information by calculating the cumulative sum of the difference between the reference value and the target value of the observation measures [Liu 2020; He 2021; Toennesen 2021]. Therefore, the fluctuation could be avoided in the process of surgeons learning a new operation, and the degree of mastery of the operation could be more accurately judged. CUSUM method gradually has become an objective evaluation standard in the field of surgical medicine, which is used to investigate the minimum number of surgical cases required to learn new technology [Liu 2020; Braca 2021; He 2021; Kudsi 2021; Lee 2021; Lenfant 2021; Szymczak 2021; Toennesen 2021; Verhaeghe 2021]. In this study, the learning curves of the two methods systematically were analyzed. The results of the analysis are helpful to the popularization of the methods for the treatment of TAAD. We believe this study has some reference value for the growth of young doctors in our center.

\section{CONCLUSIONS}

Our results show that the therapeutic effect of TAAD is related to the surgical method. After a certain period, the learning curve of the surgical team regarding aortic hemiarch replacement surgery has an earlier turning point and shorter learning cycle compare with total-arch replacement. For growing surgeons, half-arch replacement has better safety and effectiveness than total-arch replacement and can obtain better learning effect.

\section{REFERENCES}

Afifi RO, Sandhu HK, Leake SS, Rice RD, Azizzadeh A, CharltonOuw KM, et al. 2016. Determinants of Operative Mortality in Patients With Ruptured Acute Type A Aortic Dissection. Ann Thorac Surg. 101(1):64-71.

Braca P, Gaglione D, Marano S, Millefiori LM, Willett P, Pattipati K. 2021. Quickest Detection of COVID-19 Pandemic Onset. IEEE Signal Process Lett. 28:683-687.

Braverman AC. 2011. Aortic dissection: prompt diagnosis and emergency treatment are critical. Cleve Clin J Med. 78(10):685-696.

DeBakey ME, Beall AC Jr, Cooley DA, Crawford ES, Morris GC Jr, Garrett HE, et al. 1966. Dissecting aneurysms of the aorta. Surg Clin North Am. 46(4):1045-1055.

DeBakey ME, Henly WS, Cooley DA, Morris GC Jr, Crawford ES, 
Beall AC Jr. 1965. Surgical Management of Dissecting Aneurysms of the Aorta. J Thorac Cardiovasc Surg. 49:130-149.

DeBakey ME, McCollum CH, Crawford ES, Morris GC Jr, Howell J, Noon GP, et al. 1982. Dissection and dissecting aneurysms of the aorta: twenty-year follow-up of five hundred twenty-seven patients treated surgically. Surgery. 92(6):1118-1134.

Di Eusanio M, Tan ME, Schepens MA, Dossche KM, Di Bartolomeo R, Pierangeli A, et al. 2003. Surgery for acute type A dissection using antegrade selective cerebral perfusion: experience with 122 patients. Ann Thorac Surg. 75(2):514-519.

He T, Sun X, Yang Y, Yuan M, Yang G, Cheng K, et al. 2021. Learning curve for total thoracoscopic lobectomy for treating pediatric patients with congenital lung malformation. Asian J Surg.

Kato M, Ohnishi K, Kaneko M, Ueda T, Kishi D, Mizushima T, et al. 1996. New graft-implanting method for thoracic aortic aneurysm or dissection with a stented graft. Circulation. 94(9 Suppl):II188-193.

Kazui T, Yamashita K, Washiyama N, Terada H, Bashar AH, Suzuki T, et al. 2002. Impact of an aggressive surgical approach on surgical outcome in type A aortic dissection. Ann Thorac Surg. 74(5):S1844-1847; discussion S1857-1863.

Kim JB, Chung CH, Moon DH, Ha GJ, Lee TY, Jung SH, et al. 2011. Total arch repair versus hemiarch repair in the management of acute DeBakey type I aortic dissection. Eur J Cardiothorac Surg. 40(4):881-887.

Kudsi OY, Gokcal F, Bou-Ayash N, Crawford AS. 2021. Learning curve of robotic transversus abdominis release in ventral hernia repair: a cumulative sum (CUSUM) analysis. Surg Endosc.

Lee D, Kang ML, Christie L, Lim WW, Tay DX, Patel S, et al. 2021. Improving trauma care in exsanguinating patients with CHOP (critical haemorrhage to operating-room patient) resuscitation protocol-A cumulative summation (CUSUM) analysis. Injury. 52(9):2508-2514.

Lenfant L, Corrigan D, Beksac AT, Schwen Z, Kaouk J. 2021. Learning curve analysis of single-port robot-assisted extraperitoneal prostatectomy using the cumulative sum (CUSUM) method. BJU Int.

Li F, Liu N, Dong P, Hou XT. 2013. [Analysis of factors related to acute renal failure post deep hypothermia circulatory arrest surgery of type A aorta dissection surgery]. Zhonghua Wai Ke Za Zhi. 51(12):1094-1098.

Li Y, Zhang N, Xu S, Fan Z, Zhu J, Huang L, et al. 2020. Acute type A aortic intramural hematoma and type A aortic dissection: correlation between the intimal tear features and pathogenesis. Quant Imaging Med Surg. 10(7):1504-1514.

Liu H, Liu S, Zaki A, Wang X, Cong S, Yang Y, et al. 2020. Quantifying the learning curve of emergent total arch replacement in acute type A aortic dissection. J Thorac Dis. 12(8):4070-4081.

Luo C, Qi R, Zhong Y, Chen S, Liu H, Guo R, et al. 2021. Early and Long-Term Follow-Up for Chronic Type B and Type Non-A Non-B Aortic Dissection Using the Frozen Elephant Trunk Technique. Front Cardiovasc Med. 8:714638.

Ma WG, Zhu JM, Zheng J, Liu YM, Ziganshin BA, Elefteriades JA, et al. 2013. Sun's procedure for complex aortic arch repair: total arch replacement using a tetrafurcate graft with stented elephant trunk implantation.
Ann Cardiothorac Surg. 2(5):642-648.

Moon MR. 2009. Approach to the treatment of aortic dissection. Surg Clin North Am. 89(4):869-893, ix.

Pompilio G, Spirito R, Alamanni F, Agrifoglio M, Polvani G, Porqueddu M, et al. 2001. Determinants of early and late outcome after surgery for type A aortic dissection. World J Surg. 25(12):1500-1506.

Poon SS, Theologou T, Harrington D, Kuduvalli M, Oo A, Field M. 2016. Hemiarch versus total aortic arch replacement in acute type A dissection: a systematic review and meta-analysis. Ann Cardiothorac Surg. 5(3):156-173.

Sherifova S, Holzapfel GA. 2019. Biomechanics of aortic wall failure with a focus on dissection and aneurysm: A review. Acta Biomater. 99:1-17.

Sun L, Qi R, Zhu J, Liu Y, Zheng J. 2011. Total arch replacement combined with stented elephant trunk implantation: a new "standard" therapy for type a dissection involving repair of the aortic arch. Circulation. 123(9):971-978.

Szymczak P, Grzybowska ME, Sawicki S, Wydra DG. 2021. Laparoscopic Pectopexy-CUSUM Learning Curve and Perioperative Complications Analysis. J Clin Med. 10(5).

Tamai K, Hori D, Yuri K, Yamaguchi A. 2020. Additional frozen elephant trunk as a bailout for a misdeployed frozen elephant trunk in the false lumen in a patient with acute aortic dissection. Eur J Cardiothorac Surg. 57(2):399-401.

Toennesen LL, Vindum HH, Risom E, Pulga A, Nessar RM, Arshad A, et al. 2021. Learning Curves for Electromagnetic Navigation Bronchoscopy Using CUSUM Analysis. J Bronchology Interv Pulmonol.

Ueda T, Shimizu H, Hashizume K, Koizumi K, Mori M, Shin H, et al. 2003. Mortality and morbidity after total arch replacement using a branched arch graft with selective antegrade cerebral perfusion. Ann Thorac Surg. 76(6):1951-1956.

Verhaeghe C, El Hachem H, Inchboard L, Corroenne R, Dreux C, Jeanneteau P, et al. 2021. Assessment of operator performance during oocyte retrievals: residents' learning curve and continuous monitoring of senior physicians. BMC Med Educ. 21(1):193.

Wang Z, Ge M, Chen C, Lu L, Zhang L, Wang D. 2021. Hepatic dysfunction in patients who received acute DeBakey type I aortic dissection repair surgery: incidence, risk factors, and long-term outcomes. J Cardiothorac Surg. 16(1):296.

Wang Z, Zhuang X, Chen B, Wen J, Peng F, Liu X, et al. 2020. 99-Case Study of Sporadic Aortic Dissection by Whole Exome Sequencing Indicated Novel Disease-Associated Genes and Variants in Chinese Population. Biomed Res Int. 2020:7857043.

Zhou C, Li Y, Yan Y, Feng D, Wei M, Wen J. 2021. Changes in Coagulation and Fibrinolysis Systems During the Perioperative Period of Acute Type A Aortic Dissection. Heart Surg Forum. 24(2):E223-E230.

Zhou X, Krishnan A, Hsu J, Burns A, Mandal K. 2019. Delayed Surgical Management of Type A Intramural Hematoma Is Not Associated with Worse Outcomes Than Emergent Operation. Heart Surg Forum. 22(2):E103-E106. 